\title{
Variants in relation to clinical manifestation of rabies in dogs ${ }^{\star}$
}

A. Shruthi ${ }^{1 *}$, K. S. Prasanna ${ }^{2}$, Sachin 1 , M. Pradeep ${ }^{2}$, P. Hamza ${ }^{2}$, R. Anoopraj ${ }^{2}$, F. Ansar', A. M. Anagha ${ }^{1}$, M. T. K. Alin ${ }^{1}$, T. Dhanya ${ }^{1}$, V. Elizabeth ${ }^{1}$ and J. G. Ajith ${ }^{3}$ Department of Veterinary Pathology, College of Veterinary and Animal Sciences, Pookode-673576 Kerala Veterinary and Animal Sciences University, Kerala, India

Citation: Shruthi, A., Prasanna, K. S., Sachin, Hamza, P., Anoopraj, R., Ansar, F., Anagha, A. M., Alin, M. T. K., Dhanya, T., Elizabeth, V., Ajith, J. G. 2021. Variants in relation to clinical manifestation of rabies in dogs. J. Vet. Anim. Sci. 52(3): 298-302. DOI: https://doi.org/10.51966/jvas.2021.52.3.298-302

Received: 13.01.2021

Accepted: 31.01.2021

Published: 30.09 .2021

\section{Abstract}

Rabies is one among the most dangerous diseases reported to have a wider range of incubation period and clinical signs. The disease is manifested as furious or paralytic forms in dogs. The combined effects of virulence and pathogenicity of the virus along with host immune factors are the main variables leading to variation in manifestation of the disease. This paper reports the finding of a study conducted in rabies positive canine cases to correlate the role of pathophysiological factors in clinical manifestation of rabies. A total of 23 rabies positive cases were studied and their anamnesis collected. Male dogs less than 5 years of age constituted major proportion of rabies positive cases. Site of bite was observed as a critical factor in disease manifestation. Anti-rabies prophylactic vaccination had significant effect as none of the vaccinated animals developed furious form of rabies. But paralytic form was found in irregularly vaccinated dogs, probably due to effect of vaccination imparting partial immune response in these animals.

Keywords: Rabies, clinical manifestation, vaccination

Nervous dysfunction is observed to be a serious condition in dogs which may have multiple etiologies. Rabies is one of the deadliest diseases affecting different species of animals often showing atypical clinical signs. The actual pathogenesis and the factors responsible for different clinical manifestations still remain vague. Different pathophysiological factors of host immune system along with the viral pathogenicity and virulence influence the manifestation of the disease. Single dog at its infectious period have been reported to transmit the disease, which was manifested in different forms in different animals (Hemachudha et al., 2002). Wide variation in incubation period and duration of illness along with absence of any typical signs until death, impart the disease a dangerously vigorous version. Correlating different variants with the clinical manifestation of rabies therefore could help to delineate the role of the same in pathogenesis of disease. Direct fluorescent antibody technique (d-FAT) has been recommended by the WHO as the gold standard diagnostic method for rabies and is found to be highly specific and sensitive test.

*Part of the MVSc. thesis of the first author submitted to Kerala Veterinary and Animal Sciences University, Pookode, Wayanad, Kerala.

1. MVSc Scholar

** corresponding author E-mail: shruthiaorb60@gmail.com; Phone number: 9400841660

2. Assistant Professor

3. Professor and Head

Copyright: (C) 2021 Shruthi et al. This is an open access article distributed under the terms of the Creative Commons Attribution 4.0 International License (http://creativecommons.org/licenses/by/4.0/), which permits unrestricted use, distribution, and reproduction in any medium, provided the original author and source are credited. 
In the present study the animal owners were provided with the preformed questionnaire to collect the details on preexposure anti rabies vaccination, bite history, clinical signs, duration of incubation and duration of illness exhibited by the rabid animal. Brain samples from dog carcasses presented for post-mortem examination to Department of Veterinary Pathology during the period of 2019 January to 2020 February, with a clinical history of neurological abnormalities were screened for rabies using d-FAT. Twenty-three brain samples diagnosed rabies positive and six samples absent for rabies viral antigen (control group) were collected and considered for the study. Direct Fluorescent Antibody test was conducted using Fluorescein iso thiocyanate (FITC) conjugated rabies virus nucleocapsid monoclonal antibody (Merck, Germany) on impression smears from cerebrum, cerebellum, hippocampus and brain stem as per the standard protocol for FAT (Dean, 1996) and observed under fluorescent microscope (Zeiss, Progress C5).

The rabies positive cases were segregated as furious and paralytic based on the clinical signs reported. The signs exhibited in rabies were highly variable from individual to individual. Based on the diversity of signs exhibited in patient to patient, the disease was described as ' the abnormal becomes typical...' in this disease (Hanlon et al., 2007).

In both the forms, anorexia and weakness was a constant sign. When dropped jaw with salivation (64 per cent) and paraplegia/ quadriplegia (36 per cent) were the predominant signs in paralytic form, excited nature and biting without provocation with vocal changes were the noticeable changes in furious form of rabies (100\%). In a comparative study of two forms of rabies conducted by Mitrabhakdi et al. (2005), where limbic signs dominated in the furious form, paralysis of lower motor neuron was prominent in the paralytic form of rabies. Hanlon et al. (2007) observed that facial asymmetry, rolling tongue, drooling and drooping of lower jaw and severe paralysis were the important notable signs reported in case of dumb/paralytic form of the disease without any signs of aggression.
They also reported the remarkable signs of furious form such as hyperesthesia to auditory, visual, tactile stimuli and unprovoked agitation towards animate and inanimate objects in the affected animal. In all the cases of paralytic rabies, generalised weakness progressing from the limbs to the head and culminating in the respiratory distress to the animal was noted by the dog owners which were also observed by Lackay et al. (2008).

In general, there were three phases in the course of rabies infection, viz. prodromal phase, furious phase and paralytic phase. Lackay et al. (2008) suggested the possibility of overlapping of phases in rabies and occasional bypassing of the furious phase in some of the cases.

An equal proportion of two forms (48\% paralytic cases \& $52 \%$ furious cases) of rabies were encountered during the time period of sample collection inclusive of the stray animal population, otherwise in pet dogs the number of paralytic cases predominated. This finding was contradictory to the observation made by Hemachudha et al. (2013) in human rabies, where he found two third of cases to be of furious nature. Some of the variables were analysed and correlated with the variation in clinical manifestation of the disease.

The data regarding anamnesis and clinical history was collected from the dog owners using a preformed questionnaire. Out of the 23 rabies positive cases 61 per cent were male dogs and 39 per cent were female dogs. Widdowson et al. (2002) also reported that twice the number male dogs were prone to rabies than female dogs. The physiological behavioural changes in dogs during the breeding season may also be an important factor in this aspect.

Forty eight per cent of the rabies positive dogs were between one to five years of age while only seventeen per cent were below one year of age as reviewed by Widdowson et al. (2002); the age of population affected was crucial and followed a cyclic pattern whenever there is a decline in herd immunity and reduced vaccine coverage. 
Stray dogs accounted for thirty-five per cent of positive cases, all of which were reportedly showing furious form of the disease and killed by people due to the aggressive behavior. 73 per cent of pet animals showed paralytic form of the disease. Rabies virus was well adapted to host species which manifested behavioural changes favouring transmission of disease, although all the mammalian species are susceptible and are dead end hosts (Hanlon et al., 2007). From the available data it was evident that stray dogs played an important role in dissemination of rabies to the pet animal as well as human population (Prasanna, 2012). The other dog breeds positive for rabies in the collected sample included German shepherd $(21.7 \%)$, Labrador retriever (21.7\%), Doberman (4.3\%) and non-descript dogs (17.4\%),

Among the pet dogs positive for rabies no bite history was reported in 53.3 per cent of animals. Hemachudha et al. (2002) suggested that mere absence of a bite history or exposure to a rabid dog, could not exclude the chance for rabies in an endemic area. India as a whole and Kerala is much prone and endemic to rabies (Shyam, 2019). One case among the paralytic group was reported to have the possibility of mongoose bite, since the owner frequently encountered mongoose near the kennel. Sudarshan et al. (2007) reported 3.5 percent chance of rabies occurrence through bites from wild animals.

Site of bite was the other important factor in the development of disease and its form. In this study 50 per cent of paralytic group of animals with known history had bite marks on head, neck and dorsum of the body and the other 50 per cent on the hind limbs and tail of the body, while all the known cases of furious form had bite marks on the head, neck and the forelimbs. In a study conducted by Mitrabhakdi et al. (2005), 65 per cent of rabies cases with the clinical manifestation of paralytic form had bite marks on the hind limb or distal extremities of the body, while more than 65 per cent of furious form rabies cases were encountered in patients bitten on their face or fore limb by a rabid animal. Hemachudha et al. (2002) commented that bite injury from a rabid animal on head, neck, face and hands associated with bleeding were having higher risk and short term of incubation period.

Brown et al. (2016) pointed out that the disease was highly communicable when there was periodic shedding of virus through the saliva. Asaye and Getachew (2014) observed that rabies virus travelled at a fairly constant rate from peripheral nerves to brain with an average speed of $8-20 \mathrm{~mm}$ per day, depending on the site of inoculation of virus. WHO (2005) recommends proper knowledge about the management of dog bite injury as the prime factor to prevent the occurrence of the disease.

Analysis of the vaccination history revealed that most of the dog keepers were ignorant about the importance of prophylactic vaccination for rabies. Out of the 11 paralytic cases only 45.5 percent had undergone regular prophylactic vaccination and in 18 per cent, vaccination was irregular, while none were vaccinated in the furious group suggestive of the effect of vaccination in manifestation of disease. In our study a Labrador of 10 years age which was exposed to stray animal attack developed paralytic form of rabies though it followed all the post-bite vaccination protocol. Tepsumethanon et al. (2016) reported that rabies infected dogs which had undergone post-bite vaccination, had a higher chance to develop paralytic form of the disease.

In the paralytic group of animals with known history, the incubation period varied from 3 weeks to 17 weeks, whereas in furious group, most of the cases being stray animal, minimum data about the incubation period could be perceived. From the available data of 2 animals the range was very wide from 1 week to 1.5 years. Hemachudha et al. (2013) reported that incubation period for rabies varied widely from seven days to six years or more with an average of one to two months. Brown et al. (2016) reviewed that the incubation period for rabies was generally in a range of 3 to 12 weeks, rarely exceeding 6 months.

Duration of illness, reported in paralytic group was ranging from 3 days to one month with an average of 10 days and in furious cases, ranged from 4 days to 10 days with an average of 6 days. In the study conducted by 
Gadre et al. (2010) average incubation period from animal bite to symptom development was two months and the duration from development of signs to death had a mean time of 11 days. Shuangshoti et al. (2016) reported that median survival time of rabid dogs was 4 days, with 25 per cent dying within 48 hours, and usually lapsing into coma 12 hours before death.

\section{Summary}

There was an equal chance in rabies infected animal to manifest furious or paralytic form of rabies, as we could encounter equal number of positive cases of both form of rabies during the period of research. Though wide range of clinical signs were exhibited by the animals infected, dropped jaw, salivation occasionally followed by generalised paralysis or quadriplegia were the predominant signs in paralytic form. Aggressive behaviour and vocal changes were notable changes in the furious form of rabies. Young animals, less than 5 years were found more susceptible to the disease whenever there was a decline in immunity due to reduced vaccine coverage in the population. Bite from an infected animal in the regions near to the CNS (face, neck etc.) is more prone to be manifested as furious form. Prophylactic vaccination played a significant role in manifestation of the disease as paralytic form in infected animal, suggestive of a partial immune response imparted by the same. Incubation period for paralytic rabies ranged from 21 to 42 days, while 1 week to 17 months in case of furious from. Duration of illness was also seen extending from 3 days up to a month in one of the rabies cases.

\section{Acknowledgement}

The authors duly acknowledge the College of Veterinary and Animal Sciences, Pookode and Department of Veterinary Pathology for the facilities provided.

\section{Conflict of interest}

The authors declare that they have no conflict of interest.

\section{References}

Asaye, M. and Getachew, N. 2014. Rabies virus proteins and their mechanism of pathogenecity. Indian j. drugs. 2: 89-95.

Brown, C.M., Slavinski, S., Ettestad, P., Sidwa, T.J.andSorhage, F.E.2016.Compendium of animal rabies prevention and control. J. Am. Vet. Med. Assoc. 248: 505-517.

Dean, D.J. 1996. The fluorescent antibody tests. Laboratory techniques in rabies. World Health Organization, Geneva, 88p.

Gadre, G., Satishchandra, P., Mahadevan, A., Suja, M.S., Madhusudana, S.N., Sundaram, C. and Shankar, S.K. 2010. Rabies viral encephalitis: clinical determinants in diagnosis with special reference to paralytic form. J. Neurol. Neurosurg. Psychiatry. 81: 812-820.

Hanlon, C.A., Niezgoda, M. and Rupprecht, C.E. 2007. Rabies. (2 ${ }^{\text {nd }}$ edition). Academic Press. Philadelphia, 231-235 pp.

Hemachudha, T., Laothamatas, J. and Rupprecht, C.E. 2002. Human rabies: A disease of complex neuropathogenetic mechanisms and diagnostic challenges. Lancet Neurol. 1: 101-109.

Hemachudha, T., Ugolini, G., Wacharapluesadee, S., Sungkarat, W., Shuangshoti, S. and Laothamatas, J. 2013. Human rabies: Neuropathogenesis, diagnosis, and management. Lancet Neurol. 12: 498513.

Lackay, S.N., Kuang, Y. and Fu, Z.F. 2008. Rabies in small animals. Vet. Clin. North Am. 38: 851-861.

Mitrabhakdi, E., Shuangshoti, S., Wannakrairot, P., Lewis, R.A., Susuki, K., Laothamatas, J. and Hemachudha, T. 2005. Difference in neuropathogenetic mechanisms in human furious and paralytic rabies. J. Neurol. Sci. 238: 3-10.

Prasanna, K. S. 2012. Pathology of the nervous system in dogs with special reference 
to rabies. Ph.D thesis, Kerala Veterinary and Animal Sciences University, Pookode, 117p.

Shuangshoti, S., Thorner, P.S., Teerapakpinyo, C., Thepa, N., Phukpattaranont, P., Intarut, N., Lumlertdacha, B., Tepsumethanon, V. and Hemachudha, T. 2016. Intracellular spread of rabies virus is reduced in the paralytic form of canine rabies compared to the furious form. PLOS Negl. Trop. Dis. 10: 47454748.

Shyam, S. 2019. Molecular detection and histopathological studies of common viral diseases affecting nervous system of dogs and cats. M.V.Sc thesis, Kerala Veterinary and Animal Sciences University, Pookode, 74p.

Sudarshan, M.K., Madhusudana, S.N., Mahendra, B.J., Rao, N.S.N., Narayana,
D.A., Rahman, S.A., Meslin, F.X., Lobo, D. and Ravikumar, K. 2007. Assessing the burden of human rabies in India: Results of a national multi-center epidemiological survey. Int. J. Infect. Dis. 11:29-35.

Tepsumethanon, V., Likitsuntonwong, W., Thorner, P.S. and Shuangshoti, S. 2016. Dogs that develop rabies postvaccination usually manifest the paralytic subtype. Prev. Vet. Med. 131: 64-66.

Widdowson, M.A., Morales, G.J., Chaves, S. and McGrane, J. 2002. Epidemiology of urban canine rabies, Santa Cruz, Bolivia. Emerge. Infect. Dis. 8: 458-468.

World Health Organization, 2018. WHO expert consultation on rabies: third report. Vol. 1012. World Health Organization, Geneva, Switzerland, $5 p$ 